\title{
LINEARIZING CERTAIN REDUCTIVE GROUP ACTIONS
}

\author{
BY
}

H. BASS AND W. HABOUSH

\begin{abstract}
Is every algebraic action of a reductive algebraic group $G$ on affine space $C^{\prime}$ equivalent to a linear action? The "normal linearization theorem" proved below implies that, if each closed orbit of $G$ is a fixed point, then $\mathbf{C}^{n}$ is $G$-equivariantly isomorphic to $\left(\mathbf{C}^{n}\right)^{G} \times \mathbf{C}^{m}$ for some linear action of $G$ on $\mathbf{C}^{m}$.
\end{abstract}

Introduction. Let $G$ be a reductive algebraic group acting (algebraically) on affine space $\mathbf{C}^{n}$. Is the action equivalent to a linear action, i.e. is the action linear after some global polynomial change of coordinates on $\mathbf{C}^{n}$ ? This question, posed in print by Kambayashi [K, Conjecture (3.1)], motivates the present paper. The first step in the problem is to find a fixed point (to play the role of the origin for the linear action).

Fixed points are known to exist in the following cases.

1. $G$ is a finite $p$-group. (Classical; see [B-B1] for a simple algebraic proof due to Shafarevič.)

2. $G$ is a torus. (Bialynicki-Birula [B-B1] deduces this easily from case 1, using Zariski density of the $p$-torsion subgroup of $G$.)

3. $G=\mathrm{SL}_{2}$ acting on $\mathbf{C}^{n}$ without three-dimensional orbits (Panyushev $[\mathbf{P}]$ ).

Linearizability of the action has been proved in the following cases.

1. $G$ is a torus acting effectively on $\mathbf{C}^{n}$ and either

(a) $\operatorname{dim} G=n$ or $n-1$ [B-B1 and B-B2], or

(b) The action is "unmixed," in the sense that no nontrivial character, together with its inverse, occur as weights of semi-invariant polynomials, and $\operatorname{dim}\left(\mathbf{C}^{n} / G\right) \leqslant 2$ [K-R].

2. $G$ is connected semisimple and $n \leqslant 4$. The case $n=1$ is trivial. The case $n=2$ is an easy consequence of Jung's Theorem $[\mathbf{J}]$ presenting the group of polynomial automorphisms of $\mathbf{C}^{2}$ as an amalgamated free product (cf. [Sh]). The case $n=3$ is due to Kraft and Popov [KP], and the case $n=4$ to D. I. Panyushev [P]. The latter results are recent, and make heavy use of Luna's Étale Slice Theory [L]. They are the most penetrating results to date.

The linearization problem subsumes the "cancellation problem" for cartesian factors of $\mathbf{C}^{n}$ : If $Y \times \mathbf{C}^{m} \cong \mathbf{C}^{n}$, must the variety $Y$ be isomorphic to $\mathbf{C}^{n-m}$ ? This is known to be so only for $n-m \leqslant 2$ (cf. [Ru]). Suppose however that $G$ is a reductive group with a linear representation on $\mathbf{C}^{m}$ fixing only the origin. Then $G$ acts on

Received by the editors July 9, 1984.

1980 Mathematics Subject Classification. Primary 13B10, 14L17; Secondary 13F20, 20G05, 13 C10. 
$Y \times \mathbf{C}^{m}$ by $g(y, z)=(y, g z)\left(g \in G, y \in Y, z \in \mathbf{C}^{m}\right)$ and we have $\left(Y \times \mathbf{C}^{m}\right)^{G}=$ $Y \times\{0\} \cong Y$. If, viewed as an action on $\mathbf{C}^{n}\left(\cong Y \times \mathbf{C}^{m}\right)$, this action can be linearized, then $Y$ must be isomorphic to the linear space $\left(\mathbf{C}^{n}\right)^{G}$, whence $Y \cong \mathbf{C}^{n-m}$. Conversely if $Y \cong \mathbf{C}^{n-m}$, then the action can clearly be linearized.

Our main result, the "Normal Linearization Theorem" (Theorem (9.1)), is a generalization to arbitrary reductive groups of the Kambayashi-Russell result on "unmixed" torus actions, cited above. The appropriate interpretation of unmixedness is what we call a "fix pointed action" of a reductive group $G$ on an affine scheme $X$, namely an action for which $\pi: X \rightarrow X / G$ induces an isomorphism $X^{G} \rightarrow X / G$. Assuming this plus a smoothness condition on $X^{G} \subset X$ we conclude that $\pi: X \rightarrow X / G$ admits the structure of a $G$-vector bundle; in particular the fibers are linear with linear $G$-action.

Supposing that $X$ is an affine space, or, more generally (by the Quillen-Suslin proof of the Serre Conjecture) that vector bundles on $X$ are trivial, we conclude further that $X$ is $G$-isomorphic to $(X / G) \times W$, where $W$ is some rational $G$-module with $W^{G}=0$. The action is then linearizable iff the variety $X / G$ is an affine space, as in the cancellation problem discussed above.

The Normal Linearization Theorem is actually formulated for situations more general than fix pointed actions. Moreover we make some observations en route on the structure of $G$-vector bundles on affine $G$-schemes $X$. Some of these are related to results of Richardson [R1 and R2], though our proofs are different and, we feel, somewhat easier.

A final section explains how the present results might be used to approach the Jacobian Conjecture $\left[\mathbf{B C W}\right.$, stating that polynomial endomorphisms of $\mathbf{C}^{n}$ with Jacobian determinant 1 are invertible.

1. The affine quotient $\pi: X \rightarrow X / G$. We fix an algebraically closed field $k$ of characteristic zero. By a scheme $X$ we shall mean a scheme of finite type over $k$. If $X$ is affine its affine algebra will be denoted $A_{X}$, so that $X=\operatorname{Spec}\left(A_{X}\right)$.

Let $G$ be an algebraic group (over $k$ ); all such groups will be understood to be affine. We shall of ten confuse $G$ with its group $G(k)$ of rational points over $k$. A scheme $X$ together with an action of $G, G \times X \rightarrow X$, will be called a $G$-scheme.

Let $X$ be an affine $G$-scheme. Suppose that the ring $A_{X}^{G}$ of $G$-invariants is finitely generated over $k$. Classical invariant theory (cf. [Sp]) guarantees this whenever the group $G$ is reductive. Then we put $X / G=\operatorname{Spec}\left(A_{X}^{G}\right)$ and call $\pi=\pi_{X, G}: X \rightarrow X / G$ (induced by $A_{X}^{G} \subset A_{X}$ ) the affine quotient of the $G$-scheme $X$.

An orbit $G x$ is open in its closure $\overline{G x}$, which consists of $G x$ plus lower dimensional orbits. In particular an orbit of minimal dimension in $\overline{G x}$ is closed, so nonempty closed $G$-stable subsets of $X$ contain closed orbits. Equivalently: An open $G$-stable subset of $X$ containing all closed orbits is all of $X$. Part of the philosophy of this paper is to develop the latter as the geometric form of an "Equivariant Nakayama Lemma" (see $\$ \S 5$ and 6).

Suppose that $G$ is reductive. If $C_{0}, C_{1}$ are disjoint closed $G$-stable subschemes of $X$, then there is an $f \in A_{X}^{G}$ such that $\left.f\right|_{C_{1}}=i(i=0,1)$. Consequently, each fiber 
$\pi^{-1}(\xi)(\xi \in X / G)$ contains a unique closed orbit, denoted $C(\xi)$, and

$$
\pi^{-1}(\xi)=\{x \in X \mid C(\xi) \subset \overline{G x}\} .
$$

2. $A$ - $G$-modules. Let $G$ be an (affine) algebraic group (over $k$ ). A finite dimensional $k$-module $V$ on which $G(k)$ acts $k$-linearly is called rational if, relative to a basis of $V$, the matrix entries of the representation belong to $A_{G}$. In general we understand a rational G-module to be a union of finite dimensional ones. We shall call these simply $k$-G-modules.

Let $X=\operatorname{Spec}(A)$ be an affine scheme. An action of $G$ on $X$ corresponds to a (translation) action of $G$ on $A=A_{X}$ as $k$-algebra automorphisms, making $A$ a $k$ - $G$-module as above. Conversely any such action of $G$ on $A$ defines an action on $X$.

By an $A$ - $G$-module then we mean an $A$-module $M$ which is simultaneously a $k$ - $G$-module so that

$$
s(a m)=s(a) \cdot s(m)
$$

for all $s \in G, a \in A, m \in M$. These form an abelian category. If $M$ and $N$ are $A$ - $G$-modules so also is $M \otimes_{A} N$, with $G$-action $s(m \otimes n)=s m \otimes s n$. Further the symmetric algebra $S_{A}(M)$ and exterior algebra $\Lambda_{A}(M)$ similarly become $A$ - $G$-modules. The $G$-action on $\operatorname{Hom}_{A}(M, N),(s f)(m)=s\left(f\left(s^{-1} m\right)\right)$, is rational, and so makes $\operatorname{Hom}_{A}(M, N)$ an $A$-G-module, provided that $M$ is finitely $A$-generated. Further we have

$$
\operatorname{Hom}_{A}(M, N)^{G}=\operatorname{Hom}_{A-G}(M, N) .
$$

3. Reductive groups. Let $G$ be a reductive group. Then the $k$ - $G$-modules are semisimple (cf. [N, Chapter VI]). Let $\Lambda$ denote the set of classes of simple $k$ - $G$-modules, and, for $\lambda \in \Lambda$, let $V_{\lambda}$ be a simple $k$-G-module of type $\lambda$.

Let $M$ be a $k$ - $G$-module. Then

$$
M=\bigoplus_{\lambda \in \Lambda} M_{\lambda},
$$

where $M_{\lambda}$ is the isotypic component of type $\lambda$. Put $H_{\lambda}(M)=\operatorname{Hom}_{k}\left(V_{\lambda}, M\right)$, and $H_{\lambda}^{G}(M)=H_{\lambda}(M)^{G}=\operatorname{Hom}_{k-G}\left(V_{\lambda}, M\right)$. The canonical (evaluation) homomorphism

$$
\begin{aligned}
\varepsilon_{\lambda, M}: H_{\lambda}^{G}(M) \otimes_{k} V_{\lambda} & \rightarrow M_{\lambda} \\
\varepsilon_{\lambda, M}(h \otimes v) & =h(v)
\end{aligned}
$$

is an isomorphism of $k$ - $G$-modules (with trivial $G$-action on $H_{\lambda}^{G}(M)$ ). This reduces, by additivity, to the case $M=V_{\lambda}$, where it follows from Schur's Lemma (since $k$ is algebraically closed).

Now suppose that $G$ acts on $X=\operatorname{Spec}(A)$ and that $M$ is an $A$-G-module. Then $H_{\lambda}(M)=\operatorname{Hom}_{k}\left(V_{\lambda}, M\right)$ is an $A$-G-module, finitely $A$-generated if $M$ is so, $H_{\lambda}^{G}(M)$ is an $A^{G}$-module (with trivial $G$-action), and $\varepsilon_{\lambda, M}: H_{\lambda}^{G}(M) \otimes_{k} V_{\lambda} \rightarrow M_{\lambda}$ is an $A^{G}$-G-isomorphism.

(3.1) THEOREM (FINITE MULTIPLICITY). Let $G$ be a reductive group acting on the affine scheme $X=\operatorname{Spec}(A)$. Let $M$ be an $A-G$-module which is finitely A-generated. For each $\lambda \in \Lambda, H_{\lambda}^{G}(M)=\operatorname{Hom}_{k-G}\left(V_{\lambda}, M\right)$ and $M_{\lambda} \cong H_{\lambda}^{G}(M) \otimes_{k} V_{\lambda}$ are finitely generated $A^{G}$-modules. 
Proof. Form the symmetric algebra $S=S_{A}(M)=S_{0} \oplus S_{1} \oplus S_{2} \oplus \cdots \quad\left(S_{0}=\right.$ $\left.A, S_{1}=M\right)$. It is a finitely generated $k$-algebra on which $G$ acts rationally as graded algebra automorphisms. By classical invariant theory (cf. [Sp, Theorem 2.4.9]) $S^{G}$ is a finitely generated $k$-algebra. Since $S^{G}=A^{G} \oplus M^{G} \oplus S_{2}^{G} \oplus \cdots$ it follows easily that $M^{G}$ is a finitely generated $A^{G}$-module. Applying this to $H_{\lambda}(M)=$ $\operatorname{Hom}_{k}\left(V_{\lambda}, M\right)$, which is a finitely $A$-generated $A$-G-module, in place of $M$, we conclude that $H_{\lambda}^{G}(M)$ is finitely $A^{G}$-generated, whence likewise for $M_{\lambda} \cong H_{\lambda}^{G}(M)$ $\otimes_{k} V_{\lambda}$.

(3.2) Corollary. If the reductive group $G$ acts on the affine scheme $X=\operatorname{Spec}(A)$, then for each $\lambda \in \Lambda, A_{\lambda}$ is a finitely generated $A^{G}$-module.

Apply the theorem to $M=A$.

(3.3) Remarks. Let $\xi \in X / G$ correspond to the maximal ideal $m \subset A^{G}$. Then it is clear that $H_{\lambda}(A / m A)=H_{\lambda}(A) / m H_{\lambda}(A)$, and, by semisimplicity of $k-G$-modules, $H_{\lambda}^{G}(A / m A)=H_{\lambda}^{G}(A) / m H_{\lambda}^{G}(A)$. Thus the (finite) multiplicity of $V_{\lambda}$ in $A_{\pi^{-1}(\xi)}=$ $A / m A$ is

$$
m_{\lambda}(\xi)=\operatorname{dim}_{k}\left(H_{\lambda}^{G}(A) / m H_{\lambda}^{G}(A)\right) .
$$

In case $H_{\lambda}^{G}(A)$ is a flat, hence (by (3.2)) projective, $A^{G}$-module, then $m_{\lambda}(\xi)$ is a locally constant function of $\xi$, hence constant if $X / G$ is connected. In this case $m_{\lambda}=m_{\lambda}(\xi)$ can be computed on any convenient fiber $\pi^{-1}(\xi)$.

Suppose that a fiber $\pi^{-1}(\xi)$ is a (reduced) closed orbit $G x \cong G / H$, where $H=G_{x}$. Then $A / m A$ is $G$-isomorphic to $A_{G / H}=\left(A_{G}\right)^{H}$, where $H$ acts on $A_{G}$ via right translation on $G$. Now as a $G \times G$-module, via left and right translations, $A_{G} \cong$ $\bigoplus_{\lambda \in \Lambda}\left(V_{\lambda} \otimes_{k} V_{\lambda}^{*}\right)$, where $(s, t) \in G \times G$ acts on $V_{\lambda} \otimes_{k} V_{\lambda}^{*}$ as $\rho_{\lambda}(s) \otimes \rho_{\lambda}^{*}(t)$. Moreover, $V_{\lambda} \otimes V_{\lambda}^{*}$ is, for left translation, the $\lambda$-isotypic component $\left(A_{G}\right)_{\lambda}$ of $A_{G}$. Thus we have $\left(A_{G / H}\right)_{\lambda} \cong\left(V_{\lambda} \otimes_{k} V_{\lambda}^{*}\right)^{H}=V_{\lambda} \otimes_{k}\left(V_{\lambda}^{*}\right)^{H}$, so the multiplicity of $V_{\lambda}$ in $A_{G / H}$ is

$$
m_{\lambda}(G / H)=\operatorname{dim}_{k}\left(V_{\lambda}^{*}\right)^{H},
$$

where $\left(V_{\lambda}^{*}\right)^{H}=\operatorname{Hom}_{k-H}\left(V_{\lambda}, k\right)$.

4. Projective $A$-G-modules. Consider a reductive group $G$ acting on an affine scheme $X=\operatorname{Spec}(A)$.

(4.1) Proposition ("Splitting Lemma”). Let

$$
0 \rightarrow M^{\prime} \rightarrow M \stackrel{p}{\rightarrow} M^{\prime \prime} \rightarrow 0
$$

be an exact sequence of $A$-G-modules with $M^{\prime \prime}$ finitely $A$-generated. If (*) splits $A$-linearly, then it splits $A$-G-linearly.

Proof. Since (*) splits $A$-linearly,

$$
\operatorname{Hom}_{A}\left(M^{\prime \prime}, M\right) \stackrel{p \circ-}{\rightarrow} \operatorname{Hom}_{A}\left(M^{\prime \prime}, M^{\prime \prime}\right) \rightarrow 0
$$


is exact. Since $M^{\prime \prime}$ is finitely $A$-generated the latter are $A$ - $G$-modules, hence semisimple $G$-modules since $G$ is reductive. Thus we obtain a still exact sequence of $G$-invariants.

$$
\operatorname{Hom}_{A-G}\left(M^{\prime \prime}, M\right) \stackrel{p \circ}{\rightarrow} \operatorname{Hom}_{A-G}\left(M^{\prime \prime}, M^{\prime \prime}\right) \rightarrow 0
$$

Lifting $1_{M^{\prime \prime}}$ to $\operatorname{Hom}_{A-G}\left(M^{\prime \prime}, M\right)$ gives the desired splitting of $(*)$.

(4.2) Corollary. Let $P$ be a finitely A-generated A-G-module. The following conditions are equivalent.

(a) $P$ is $A$-projective.

(b) $P$ is $A$-G-projective.

(c) $P$ is a direct summand of $V_{A} \stackrel{\text { def }}{=} A \otimes_{k} V$ for some finite dimensional $k$ - $G$-module $V$.

Proof. Let $V$ be a $k$ - $G$-module and $M$ an $A$ - $G$-module. The natural isomorphism $\operatorname{Hom}_{k}(V, M) \cong \operatorname{Hom}_{A}\left(V_{A}, M\right)$ is an $A$-G-isomorphism, whence $\operatorname{Hom}_{A-G}\left(V_{A}, M\right) \cong$ $\operatorname{Hom}_{k-G}(V, M)$. Since $k$-G-modules are semisimple, $\operatorname{Hom}_{k-G}(V,-)$ is exact, hence $\operatorname{Hom}_{A-G}\left(V_{A},-\right)$ is exact, so $V_{A}$ is $A-G$-projective, whence (c) $\Rightarrow$ (b). Since $V_{A}$ is $A$-free, (c) $\Rightarrow$ (a).

Choose in $P$ a finite dimensional $k$ - $G$-module $V$ that generates $P$ as an $A$-module. The inclusion $V \subset P$ defines an $A$-G-homomorphism $V_{A} \rightarrow P$ that is surjective. Assuming (b), it splits, whence (b) $\Rightarrow$ (c). Assuming (a) also, it splits, by the splitting lemma, whence $(a) \Rightarrow(c)$.

(4.3) TheOREM. Let $M$ be a finitely A-generated $A-G$-module. The following conditions are equivalent.

(a) $M$ is $A^{G}$-flat.

(b) $M$ is $A^{G}$-projective.

(c) $M$ is $A^{G-G \text {-projective. }}$

(d) $H_{\lambda}^{G}(M)$ is $A^{G}$-projective for each $\lambda \in \Lambda$.

Proof. An $A^{G}$ - $G$-module $M$ decomposes as

$$
M=\bigoplus_{\lambda} M_{\lambda}=\bigoplus_{\lambda} H_{\lambda}^{G}(M) \otimes_{k} V_{\lambda}
$$

with each $M_{\lambda}$ and $H_{\lambda}^{G}(M)$ finitely $A^{G}$-generated. Whence

$$
\begin{aligned}
M \text { is } A^{G} \text {-flat } & \Leftrightarrow M_{\lambda} \text { is } A^{G} \text {-flat } \forall \lambda \\
& \Leftrightarrow H_{\lambda}^{G}(M) \text { is } A^{G} \text {-flat } \forall \lambda \\
& \Leftrightarrow H_{\lambda}^{G}(M) \text { is } A^{G} \text {-projective } \forall \lambda \\
& \Leftrightarrow M_{\lambda} \text { is } A^{G} \text {-projective } \forall \lambda \\
& \Leftrightarrow M \text { is } A^{G} \text {-projective. }
\end{aligned}
$$

By Corollary (4.2), $M_{\lambda}$ is $A^{G}$-projective $\forall \lambda \Leftrightarrow M_{\lambda}$ is $A^{G}$-G-projective $\forall \lambda \Leftrightarrow M$ is $A^{G}$ - $G$-projective. Whence the theorem. 
(4.4) Corollary. Suppose that projective $A^{G}$-modules are free. If $M$ is a finitely $A$-generated $A$-G-module that is $A^{G}$-flat, then there is a $k$-G-module $W$ with finite multiplicities (i.e. $\operatorname{dim}_{k} W_{\lambda}<\infty \forall \lambda$ ) such that $M$ is isomorphic as an $A^{G}-G$-module to $A^{G} \otimes_{k} W$.

Proof. The hypotheses and the theorem imply that, for each $\lambda, H_{\lambda}^{G}(M) \cong\left(A^{G}\right)^{n_{\lambda}}$ for some $n_{\lambda} \geqslant 0$. Thus $M$, as an $A^{G}$-G-module, is isomorphic to

$$
\begin{aligned}
\bigoplus_{\lambda}\left(H_{\lambda}^{G}(M) \otimes_{k} V_{\lambda}\right) & \cong \bigoplus_{\lambda}\left(\left(A^{G}\right)^{n_{\lambda}} \otimes_{k} V_{\lambda}\right) \\
& \cong \bigoplus_{\lambda}\left(A^{G} \otimes_{k} V_{\lambda}^{n_{\lambda}}\right) \cong A^{G} \otimes_{k} W,
\end{aligned}
$$

where $W=\oplus_{\lambda} V_{\lambda}^{n_{\lambda}}$.

(4.5) Remark. In case projective $A^{G}$-modules are free and $A$ is $A^{G}$-flat, then, by (4.4), $A \cong A^{G} \otimes_{k} W$ for some $k$-G-module $W \subset A$ with finite multiplicities. Cases where this situation prevails are treated by Richardson (see [R1, and R2, Theorem B]).

5. The $G$-radical. Let $G$ be a reductive group. For any $k$ - $G$-module $V$ we have

$$
V=V^{G} \oplus \Delta_{G}(V),
$$

where $\Delta_{G}(V)$ is the sum of the nontrivial isotypic components of $V$, or, alternatively, it is the sub- $k$-module generated by all $s v-v(s \in G, v \in V)$.

Suppose that $G$ acts on the affine scheme $X=\operatorname{Spec}(A)$. Then $A=A^{G} \oplus \Delta_{G}(A)$ is a decomposition as an $A^{G}$-module. We put

$$
\begin{aligned}
\Delta_{G}^{+}(A) & =\text { the smallest ideal of } A \text { containing } \Delta_{G}(A) \\
& =\text { the ideal of } A \text { generated by } \Delta_{G}(A),
\end{aligned}
$$

and

$$
\begin{aligned}
\Delta_{G}^{-}(A) & =\text { the largest ideal of } A \text { contained in } \Delta_{G}(A) \\
& =\text { the sum of all } A \text {-ideals contained in } \Delta_{G}(A) .
\end{aligned}
$$

These are $G$-stable ideals of $A$, and

$$
\Delta_{G}^{-}(A) \subset \Delta_{G}(A) \subset \Delta_{G}^{+}(A) .
$$

Clearly $\Delta_{G}^{+}(A)$ is the smallest ideal of $A$ modulo which the action of $G$ is trivial. Hence it defines the scheme of fixed points,

$$
X^{G}=\operatorname{Spec}\left(A / \Delta_{G}^{+}(A)\right) \subset \operatorname{Spec}(A)=X .
$$

We have $\Delta_{G}^{+}(A)=\Delta_{G}^{+}(A)^{G} \oplus \Delta_{G}(A)$, so that $A / \Delta_{G}^{+}(A)=A^{G} / \Delta_{G}^{+}(A)^{G}$, and the diagram

$$
\begin{array}{ccc}
X^{G} & \subset & X \\
& \searrow & \downarrow \pi \\
& & X / G
\end{array}
$$


corresponding to

$$
\begin{aligned}
A / \Delta_{G}^{+}(A) & \leftarrow \quad A \\
& \longleftarrow \quad A^{G}
\end{aligned}
$$

identifies $X^{G}$ with a closed subscheme of $X / G$.

We now interpret $\Delta_{G}^{-}(A)$ geometrically.

If $x \in X(k)$ let $m_{x} \subset A$ denote its maximal ideal. If $s \in G$, then $s\left(m_{x}\right)=m_{s x}$. Put

$$
P_{x}=\bigcap_{s \in G} s\left(m_{x}\right)=\bigcap_{s \in G} m_{s x},
$$

the largest $G$-stable ideal contained in $m_{x}$. Then every $G$-stable proper ideal is contained in some $P_{x}$. Further $\operatorname{Spec}\left(A / P_{x}\right) \subset X$ is just the (reduced) orbit closure $\overline{G x}$. Hence if $G$ is connected, then $P_{x}$ is prime. Further we have

$$
P_{x} \subset P_{y} \Leftrightarrow \overline{G y} \subset \overline{G x} \text {. }
$$

Hence

(5.1) Proposition. $P$ is a maximal G-stable proper ideal of $A$ iff $P=P_{x}$ for some $x \in X$ such that $G x$ is closed, and then $G x=\operatorname{Spec}\left(A / P_{x}\right) \subset X$.

The $G$-radical of $A$ is, by definition,

$$
G-\operatorname{rad}(A)=\bigcap_{G x \text { closed }} P_{x} .
$$

Thus $\operatorname{Spec}(A / G-\operatorname{rad}(A))$ is the reduced subscheme corresponding to the closure of the union of the closed orbits; we shall call it briefly the "closed orbit closure". It is the largest reduced closed subscheme of $X$ on which the $G$-action is "stable" in the sense of Popov [Po]. Since $G-\operatorname{rad}(A)$ is $G$-stable we have

$$
G-\operatorname{rad}(A)=G-\operatorname{rad}(A)^{G} \oplus \Delta_{G}(G-\operatorname{rad}(A)) .
$$

(5.2) Proposition. (a) $G-\operatorname{rad}(A)^{G}=\operatorname{nil}\left(A^{G}\right)$, the nil radical of $A^{G}$.

(b) $G-\operatorname{rad}(A)=\sqrt{\Delta_{G}^{-}(A)}$, the radical of $\Delta_{G}^{-}(A)$.

(c) If $A^{G}$ is reduced, e.g. if $A$ is reduced, then $G-\operatorname{rad}(A)=\Delta_{G}^{-}(A)$.

(d) If $A^{G}$ is an integral domain and $G$ is connected, then $G-\operatorname{rad}(A)\left(=\Delta_{G}^{-}(A)\right)$ is a prime ideal.

Proof. Let $\xi \in X / G$ with maximal ideal $m_{\xi} \subset A^{G}$. Then $\pi^{-1}(\xi)=\operatorname{Spec}\left(A / m_{\xi} A\right)$. Choose $x \in C(\xi)$, the closed orbit in $\pi^{-1}(\xi)$, and write $P_{\xi}$ for $P ; P_{\xi}$ is the unique maximal $G$-stable ideal of $A$ containing $m_{\xi} A=m_{\xi} \oplus m_{\xi} \Delta_{G}(A)$. We have

$$
\begin{aligned}
G-\operatorname{rad}(A) & =\bigcap_{\xi \in X / G} P_{\xi}=\bigcap_{\xi \in X / G}\left(m_{\xi} \oplus m_{\xi} \Delta_{G}^{-}(A)\right) \\
& =\left(\bigcap_{\xi \in X / G} m_{\xi}\right) \oplus\left(\bigcap_{\xi \in X / G} m_{\xi} \Delta_{G}(A)\right) \\
& =\operatorname{nil}\left(A^{G}\right) \oplus \Delta_{G}(G-\operatorname{rad}(A)),
\end{aligned}
$$

by the Nullstellensatz (applied to $A^{G}$ ); whence (a). 
If $A^{G}$ is reduced, then $G-\operatorname{rad}(A)=\Delta_{G}(G-\operatorname{rad}(A))$ is an ideal contained in $\Delta_{G}(A)$, hence contained in $\Delta_{G}^{-}(A)$, by definition. Now (c) follows by showing that $\Delta_{G}^{-}(A) \subset$ $G-\operatorname{rad}(A)$. It suffices to show that each $P_{\xi}(\xi \in X / G)$ contains $\Delta_{G}^{-}(A)$. If not, then by maximality of $P_{\xi}$ we have $A=P_{\xi}+\Delta_{G}^{-}(A)=m_{\xi} \oplus\left(\Delta_{G}\left(P_{\xi}\right)+\Delta_{G}^{-}(A)\right) \subset m_{\xi} \oplus$ $\Delta_{G}(A)$, which is absurd; this proves (b).

To show that $G-\operatorname{rad}(A) \subset \sqrt{\Delta_{G}^{-}(A)}$ note first that both sides contain $\operatorname{nil}(A)$, which is a $G$-stable ideal. Moreover $G-\operatorname{rad}(A / \operatorname{nil}(A))=G-\operatorname{rad}(A) / \operatorname{nil}(A)$ and $\Delta_{G}^{-}(A / \operatorname{nil}(A))=\Delta_{G}^{-}(A) /\left(\Delta_{G}^{-}(A) \cap \operatorname{nil}(A)\right)$. Thus we may pass to $A / \operatorname{nil}(A)$, and reduce to the case when $A$ is reduced. Then, by (a), $G-\operatorname{rad}(A) \subset \Delta_{G}(A)$ so, by definition of $\Delta_{G}^{-}(A), G-\operatorname{rad}(A) \subset \Delta_{G}^{-}(A)$. This concludes the proof of (c).

Suppose finally that $A^{G}$ is an integral domain. Let $\Delta_{G}^{-}(A)=Q_{1} \cap \cdots \cap Q_{n}$ be the primary decomposition of $\Delta_{G}^{-}(A)(=G-\operatorname{rad}(A)$, by (c)). Since $G-\operatorname{rad}(A)$ is a radical ideal each $Q_{i}$ is prime. Since $G-\operatorname{rad}(A)$ is $G$-stable, $G$ permutes the $Q_{i}$ 's. Suppose that $G$ is connected. Then $G$ stabilizes each $Q_{i}$. Hence $Q_{i}=Q_{i}^{G} \oplus \Delta_{G}\left(Q_{i}\right)$. Then $Q_{1}^{G} \cap \cdots \cap Q_{n}^{G}=G \operatorname{rad}(A)^{G}=0$. Since $A^{G}$ is an integral domain there is an $i$ such that $Q_{i}^{G}=0$. Then $Q_{i}=\Delta_{G}\left(Q_{i}\right) \subset \Delta_{G}(A)$ so, by definition of $\Delta_{G}^{-}(A), Q_{i} \subset$ $\Delta_{G}^{-}(A)$. But then $Q_{i}=\Delta_{G}^{-}(A)$, so $\Delta_{G}^{-}(A)$ is prime.

(5.3) Proposition. Let $J$ be a $G$-stable ideal of $A$ and put $X_{0}=\operatorname{Spec}(A / J) \subset X=$ $\operatorname{Spec}(A)$. The following conditions are equivalent.

(i) $X_{0}$ contains all closed orbits in $X$.

(ii) $J \subset G-\operatorname{rad}(A)$.

(iii) $J^{G}$ is a nilpotent ideal in $A^{G}$.

The remarks preceding (5.2) show that (i) $\Leftrightarrow$ (ii), and (ii) $\Rightarrow$ (iii) by (5.2)(a). To show that (iii) $\Rightarrow$ (ii), assume that $J^{G}$ is nilpotent. Put $A_{\text {red }}=A / \operatorname{nil}(A)$ and $J_{\text {red }}=$ the image of $J$ in $A_{\text {red }}$. Then $J_{\text {red }}^{G}=0$ so $J_{\text {red }} \subset \Delta_{G}^{-}\left(A_{\text {red }}\right) \subset G-\operatorname{rad}\left(A_{\text {red }}\right)$ (by (5.2)(c)). Therefore $J$ is contained in the inverse image in $A$ of $G-\operatorname{rad}\left(A_{\text {red }}\right)$, which is $G-\operatorname{rad}(A)$.

6. Equivariant Nakayama Lemma. Let $G$ be a reductive group acting on $X=$ $\operatorname{Spec}(A)$. We fix a $G$-stable ideal, $J \subset G-\operatorname{rad}(A)$.

(6.1) Proposition ("Equivariant Nakayama Lemma”). Let $M$ be a finitely A-generated A-G-module.

(a) If $J M=M$, then $M=0$.

(b) If $N$ is an $A$-G-submodule of $M$ and if $M=N+J M$, then $M=N$.

Proof. Applying (a) to $M / N$ one obtains (b). To prove (a) recall that $J M=M$ iff $J+\operatorname{ann}_{A}(M)=A$, where $\operatorname{ann}_{A}(M)=\{a \in A \mid a M=0\}$, a $G$-stable ideal. (See [NB1, II, §4, no. 4, Corollaire de la Proposition 18].) Since $J$ is contained in every maximal $G$-stable ideal, $\operatorname{ann}_{A}(M)$ cannot be contained in a maximal $G$-stable ideal, so $\operatorname{ann}_{A}(M)=A$, i.e. $M=0$.

(6.2) Proposition. Let $P, Q$ be finitely A-generated $A$-G-modules. Assume that $P$ is A-projective.

(a) Any A-G-homomorphism $u_{0}: P / J P \rightarrow Q / J Q$ lifts to an $A$-G-homomorphism u: $P \rightarrow Q$. 
(b) If $u_{0}$ is surjective, then $u$ is surjective.

(c) If $u_{0}$ is an isomorphism and if $Q$ also is A-projective, then $u$ is an isomorphism.

Proof. (a) In the commutative diagram of $A$-G-homomorphisms

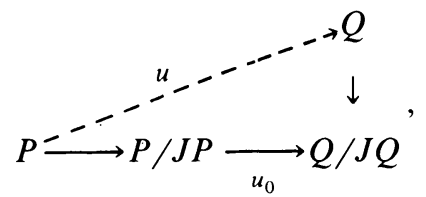

$u$ exists because $P$ is $A$ - $G$-projective (Corollary (4.2)).

(b) If $u_{0}$ is surjective and $C=\operatorname{Coker}(u)$, then $C / J C=\operatorname{Coker}\left(u_{0}\right)=0$, so $C=0$ by the Equivariant Nakayama Lemma.

(c) Suppose that $u_{0}$ is an isomorphism and that $Q$ is $A$-projective. Then $u$ is surjective, by (b), and so splits $A$-linearly (in fact $A$-G-linearly, by Corollary (4.2)). It follows that $K=\operatorname{Ker}(u)$ is a direct summand of $P$, so that $K / J K=\operatorname{Ker}\left(u_{0}\right)=0$, whence $K=0$ by Nakayama again.

(6.3) Corollary. Let $P$ be an A-G-module, finitely generated and projective as an $A$-module. Let $V$ be a finite dimensional $k$-G-module. Suppose that $P / J P$ and $V_{A / J}=(A / J) \otimes_{k} V$ are isomorphic as $(A / J)$-G-modules. Then $P$ and $V_{A}=A \otimes_{k} V$ are isomorphic as $A$-G-modules.

This follows from the proposition, part (c), with $V_{A}$ in the role of $Q$.

(6.4) Corollary. Suppose that $A=A_{0} \oplus J$ for some G-stable subalgebra $A_{0}$. Let $P$ be an $A$-G-module finitely generated and projective as an $A$-module. Then $P$ is $A$-G-isomorphic to $A \otimes_{A_{0}} P_{0}$, where $P_{0}$ is the $A_{0}-G$-module $P / J P$ (and we identify $A / J$ with $A_{0}$ ).

We apply the proposition, part (c), with $Q=Q \otimes_{A_{0}} P_{0}$, noting that $Q / J Q \cong P_{0}$.

(6.5) Geometric interpretation. Let $X_{0}=\operatorname{Spec}(A / J) \subset X=\operatorname{Spec}(A)$. The condition that $J$ is $G$-stable and contained in $G$-rad $(A)$ signifies that $X_{0}$ is $G$-stable and contains all closed orbits. Then $A$ - $G$-modules $P, Q$ define coherent $G$-sheaves $\tilde{P}, \tilde{Q}$ on $X$, with $\tilde{P}$ locally free, i.e. a " $G$-vector bundle". Their restrictions to $X_{0}$ are $\tilde{P}_{0}$, $\tilde{Q}_{0}$, where $P_{0}=P / J P, Q_{0}=Q / J Q$.

(a) Any $G$-morphism $u_{0}: \tilde{P}_{0} \rightarrow \tilde{Q}_{0}$ extends to a $G$-morphism $u: \tilde{P} \rightarrow \tilde{Q}$.

(b) If $u_{0}$ is surjective, then $u$ is surjective.

(c) If $u_{0}$ is an isomorphism and $\tilde{Q}$ also is locally free, then $u$ is an isomorphism.

7. Luna's Equivariant Zariski Main Theorem. We shall make use of the following results, quoted for reference.

(7.1) Theorem (Luna [L, I, §4]). Let $G$ be a reductive group. Let $X, Y$ be affine $G$-schemes. Let $\varphi: X \rightarrow Y$ be a G-morphism with finite fibers. Then there is a factorization

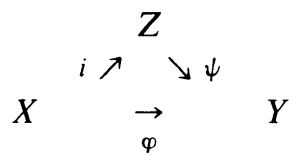


where $Z$ is an affine $G$-scheme, $i$ and $\psi$ are $G$-morphisms, $i$ is an open immersion with $i^{0}: A_{Z} \rightarrow A_{X}$ injective, and $\psi$ is a finite morphism.

(7.2) Corollary (Luna [L, I, §4]). Assume above that:

(a) $\varphi$ preserves closed orbits (i.e. Gx closed in $X \Rightarrow G \varphi(x)$ is closed in $Y$ ).

(b) $\varphi$ is quasi-finite (i.e. has finite fibers).

(c) $\varphi / G: X / G \rightarrow Y / G$ is finite.

Then $\varphi$ is finite.

8. Local complete intersections (LCI) and retracts. We fix an affine scheme $X=\operatorname{Spec}(A)$, an ideal $J \subset A, A_{0}=A / J$, and the closed subscheme $X_{0}=\operatorname{Spec}\left(A_{0}\right)$ of $X$. Consider the $A_{0}$-module $N=J / J^{2}$. The graded $A_{0}$-algebra

$$
\operatorname{gr}_{J}(A)=\bigoplus_{n \geqslant 0} J^{n} / J^{n+1}
$$

is generated by $N$ in degree 1 , so there is a canonical surjection of graded $A_{0}$-algebras

$$
\gamma: S=S_{A_{0}}(N) \rightarrow \operatorname{gr}_{J}(A) .
$$

One says that $X_{0}$ is a local complete intersection in $X$, or that $J$ is a local complete intersection ideal in $A$, if the following conditions hold:

(i) The $A_{0}$-module $N=J / J^{2}$ is projective.

(ii) $\gamma: S=S_{A_{0}}(N) \rightarrow \operatorname{gr}_{J}(A)$ is an isomorphism.

(8.1) EXAmple. A (closed) point $x \in X$ is a local complete intersection in $X$ iff $x$ is a regular (or smooth) point of $X$, i.e. its local ring $A_{m_{x}}$ is regular. (See [NB2, VIII, $\S 5$, Theorem 1].) We shall write $X_{\text {reg }}$ for the set of regular points of $X$, and call $X$ (and $A$ ) regular if $X_{\text {reg }}=X$. In general $X_{\text {reg }}$ is open in $X$ (cf. [EGA, IV, (6.12.5)]).

(8.2) Remark. Suppose that a reductive group $G$ acts on $X$. Then $X_{\text {reg }}$ is $G$-stable. If $X_{\text {reg }}$ contains all closed orbits, then, since the $G$-stable closed set $X-X_{\text {reg }}$ contains no closed orbits, it must be empty, i.e. $X$ is regular.

(8.3) Lemma. If $X_{0}$ is regular and $X_{0}$ is a local complete intersection in $X$, then $X_{0} \subset X_{\text {reg }}$.

(A background reference for this proof is [NB2, VIII, §5].)

Let $m$ be a maximal ideal of $A$ containing $J$. We must show that $A_{m}$ is regular. By assumption, $\left(A_{0}\right)_{m_{0}}=A_{m} / J_{m}$ is regular, where $m_{0}=m / J$. Further it is clear that $J_{m}$ is a local complete intersection ideal in $A_{m}$, by exactness of localization. It follows that $J_{m}$ is generated by a regular sequence $a_{1}, \ldots, a_{r}$ in $A_{m}$; in particular $\operatorname{dim}\left(A_{0}\right)_{m_{0}}=\operatorname{dim} A_{m}-r$. Since $m_{0}\left(A_{0}\right)_{m_{0}}=m A_{m} / J_{m}$ is generated by $\operatorname{dim}\left(A_{0}\right)_{m_{0}}$ elements (by regularity) it follows that $m A_{m}$ is generated by $r+\operatorname{dim}\left(A_{0}\right)_{m_{0}}=$ $\operatorname{dim} A_{m}$ elements, so $A_{m}$ is regular.

(8.4) Proposition. Suppose that $A=A_{0} \oplus J$ for some subalgebra $A_{0}$ of $A$ (i.e. $X_{0}$ is a retract of $X$ ). Then the following conditions are equivalent.

(a) $X_{0} \subset X_{\text {reg }}$.

(b) (0) $X_{0}$ is regular,

(1) $X_{0}$ is a local complete intersection in $X$. 
The lemma gives (b) $\Rightarrow$ (a). Assume (a), let $m_{0}$ be a maximal ideal of $A_{0}$, and put $m=m_{0} \oplus J$. We are given that $A_{m}$ is regular and must show that $\left(A_{0}\right)_{m_{0}}$ is regular and that $J_{m}$ is a local complete intersection ideal in $A_{m}$. We have

$$
m / m^{2}=\left(m_{0} / m_{0}^{2}\right) \oplus(J / m J)
$$

and $J / m J=J /\left(m_{0} J+J^{2}\right)=N / m_{0} N$. Choose $a_{1}, \ldots, a_{d_{0}} \in m_{0}$ to lift a $k$-basis of $m_{0} / m_{0}^{2}$ and $a_{d_{0}+1}, \ldots, a_{d} \in J$ to lift a $k$-basis of $J / m J$. Then $a_{1}, \ldots, a_{d}$ is a regular system of parameters in $A_{m}$, and, by Nakayama's Lemma, $J_{m}$ is generated by the regular sequence $a_{d_{0}+1}, \ldots, a_{d}$. Both conclusions now follow easily.

\section{The Normal Linearization Theorem.}

(9.1) THEOREM. Let $G$ be a reductive group acting on the affine scheme $X=\operatorname{Spec}(A)$. Let $X_{0}=\operatorname{Spec}(A / J)$ be a closed subscheme of $X$. Assume:

(a) $X_{0}$ is $G$-stable and contains all closed orbits.

(b) There is a G-equivariant retraction $\pi: X \rightarrow X_{0}$.

(c) $X_{0}$ is a local complete intersection in $X$.

Then $\pi: X \rightarrow X_{0}$ admits the structure of a G-vector bundle over $X_{0}$.

(9.2) Remarks. 1. Algebraically the conditions of the theorem translate as follows.

(a) $J$ is $G$-stable and $J \subset G$-rad( $A$ ).

(b) $A=A_{0} \oplus J$ for some $G$-stable subalgebra $A_{0}$.

(c) $J$ is a local complete intersection ideal in $A$.

The conclusion then is that $A$, as an $A_{0}$-algebra with $G$-action, is isomorphic to a symmetric algebra $S=S_{A_{0}}(N)$ where $N$ is an $A_{0}$-G-module finitely generated and projective as an $A_{0}$-module.

2. By Corollary (6.4), conditions (a) and (b) imply that every $G$-vector bundle on $X$ is the pullback of one on $X_{0}$.

3. Suppose that $X_{0} \subset X_{\text {reg }}$, and assume (b). Then Proposition (8.4) implies condition (c), that $X_{0}$ is a local complete intersection in $X$, as well as the regularity of $X_{0}$. The conclusion of the theorem then further implies that $X$ itself is regular.

4. Put $N=J / J^{2}$. Condition (c) means:

(c)(i) $N$ is a projective $A_{0}$-module,

(c)(ii) $\gamma: S=S_{A_{0}}(N) \rightarrow \operatorname{gr}_{J}(A)$ is an isomorphism.

Consider the exact sequence of $A_{0}$ - $G$-modules,

$$
0 \rightarrow J^{2} \rightarrow J \rightarrow N \rightarrow 0 .
$$

It splits $A_{0}$-linearly, by (c)(i), hence $A_{0}$ - $G$-linearly, by the Splitting Lemma (Proposition (4.1)). Let $u^{0}: N \rightarrow J$ be such an $A_{0}$-G-splitting. It extends to an $A_{0}$-algebra homomorphism $u^{0}: S=S_{A_{0}}(N) \rightarrow A$ which is still $G$-equivariant. The corresponding $G$-morphism $u: X \rightarrow Y=\operatorname{Spec}(S)$ will be called a $G$-linear approximation to $\left(X, X_{0}\right)$. Its existence required only conditions (b) and (c)(i). The theorem will be proved by showing that any such $G$-linear approximation is an isomorphism. 
Proof OF TheOREM (9.1). Let

$$
\begin{gathered}
X=\operatorname{Spec}(A) \stackrel{u}{\rightarrow} Y=\operatorname{Spec}(S) \\
\searrow \swarrow \\
X_{0}=\operatorname{Spec}\left(A_{0}\right)
\end{gathered}
$$

be a $G$-linear approximation to $\left(X, X_{0}\right)$, as in Remark 4 above. We first prove:

(1) $u$ is étale.

The locus in $X$ where $u$ is étale is open and $G$-stable. Hence it is all of $X$ once it contains all closed orbits. It suffices therefore to show that $u$ is étale at each $x \in X_{0} \subset X$. The maximal ideal in $A$ of $x$ has the form $m_{x}=m_{0}+J$, where $m_{0}$ is maximal in $A_{0}$. By the construction of $u_{0}: S=S_{A_{0}}(N) \rightarrow A$, and the assumption that $X_{0}$ is a local complete intersection in $X$, the map of associated graded rings

$$
\operatorname{gr}\left(u^{0}\right): \operatorname{gr}_{S_{+}}(S)=S \rightarrow \operatorname{gr}_{J}(A),
$$

where $S_{+}=N \cdot S$, is an isomorphism. Since $\bigcap_{n} S_{+}^{n}=0$ it follows that $u^{0}$ is injective; we shall view $u^{0}$ as an inclusion, $S \subset A$. The maximal ideal in $S$ of $y=u(x)$ is $m_{y}=m_{x} \cap S=m_{0} \oplus S_{+}$. We want to show that $S_{m_{x}} \rightarrow A_{m_{x}}$ is étale. It suffices to show that the induced map of completed local rings $\hat{S}_{m_{1}} \rightarrow \hat{A}_{m_{x}}$ is an isomorphism. The isomorphism $\operatorname{gr}\left(u^{0}\right)$ of associated graded rings implies an isomorphism from the $S_{+}$-adic completion $\bar{S}$ of $S$ to the $J$-adic completion of $\bar{A}$ of $A$, one which carries $m_{y} \bar{S}=m_{0} \oplus \bar{S}_{+}$to $m_{X} A=m_{0} \oplus \bar{J}$. Since $\hat{S}_{m_{\mathrm{y}}}$ is the $m_{y} \bar{S}$-adic completion of $\bar{S}$, and $\hat{A}_{m_{x}}$ is the $m_{x} \bar{A}$-adic completion of $\bar{A}$, we conclude, as desired, that $\hat{S}_{m_{\mathrm{v}}} \rightarrow \hat{A}_{m_{\text {. }}}$ is an isomorphism.

(2) The image of $X_{0}=\operatorname{Spec}\left(A_{0}\right) \stackrel{\cong}{\rightarrow} \operatorname{Spec}\left(S / S_{+}\right) \subset Y$ contains all closed orbits of $Y$. Further $u: X \rightarrow Y$ carries closed orbits in $X$ to closed orbits in $Y$.

We have $S_{+}=S \cap J$ so $S_{+}^{G} \subset J^{G}$, and $J^{G}$ is nilpotent (in $A^{G}$ ) by (5.3), hence $S_{+}^{G}$ is nilpotent, so, by (5.3) again, $S_{+} \subset G-\operatorname{rad}(S)$, i.e. $X_{0} \subset Y$ contains all closed orbits. Since $u: X \rightarrow Y$ induces the identity from $X_{0} \subset X$ to $X_{0} \subset Y$, closed orbits are preserved.

(3) $u / G: X / G \rightarrow Y / G$ is a finite morphism.

We have $S=A_{0} \oplus S_{+} \subset A=A_{0} \oplus J$, and we must show that $A^{G}=A_{0}^{G} \oplus J^{G}$ is a finitely generated module over $S^{G}=A_{0}^{G} \oplus S_{+}^{G}$. This follows because $J^{G}$, being nilpotent, is a finitely generated $A_{0}^{G}$-module.

Now since an étale morphism is quasi-finite, conclusions (1), (2) and (3) above supply the hypotheses of Luna's Equivariant ZMT (Corollary (7.2)). It follows that:

(4) $u$ is finite, hence a finite étale covering.

The degree of this covering is locally constant over $Y$. The locus in $Y$ where $u$ has degree 1 is open and $G$-stable. To show that $u$ is an isomorphism, i.e. everywhere of degree 1, it therefore suffices to show that $u$ has degree 1 over each closed orbit of $Y$. Let $G y(y \in Y)$ be a closed orbit; note that $G y \subset X_{0} \subset Y$, by (3). Since $u$ is finite, $u^{-1}(G y)$ must be a finite union of $G$-orbits of the same dimension as $G y$, hence each of them is closed. Consequently, $u^{-1}(G y) \subset X_{0} \subset X$. Since $u$ maps $X_{0} \subset X$ isomorphically to $X_{0} \subset Y, u$ has degree 1 over $X_{0} \subset Y$, hence $u$ is an isomorphism. This proves Theorem (9.1). 
10. Fix pointed actions. Let $G$ be a reductive group acting on $X=\operatorname{Spec}(A)$. Recall that $A=A^{G} \oplus \Delta_{G}(A)$.

(10.0) Definition. Call the action of $G$ on $X$ or on $A$ fix pointed if the following equivalent conditions hold.

(a) $\Delta_{G}(A)$ is an ideal, i.e. $\Delta_{G}^{-}(A)=\Delta_{G}(A)=\Delta_{G}^{+}(A)$, in the notation of $\S 5$.

(b) $\pi: X \rightarrow X / G$ induces an isomorphism $X^{G} \rightarrow X / G$.

Indeed the latter corresponds to the surjective homomorphism $A^{G} \rightarrow A / \Delta_{G}^{+}(A)$. The following equivalent conditions are an immediate consequence of (a) and (b).

(c) Every closed orbit is a point.

(d) Every nonempty closed $G$-stable subset of $X$ contains a fixed point.

(e) $\Delta_{G}^{+}(A)^{G}$ is a nilpotent ideal in $A^{G}$.

If $X / G$ is reduced, e.g. if $X$ is reduced, then all of the above conditions are equivalent (Proposition (5.2)).

We call the action one fix pointed if it is fix pointed with a unique fixed point (so that $A^{G}$ is an artin local ring).

Let $\Lambda$ denote the set of types of simple $k-G$-modules, and, for each $\lambda \in \Lambda$, let $V_{\lambda}$ be a simple $k-G$-module of type $\Lambda$.

(10.1) Proposition. Assume that $G$ acts fix pointedly on $X$. Let $P$ be an $A-G-$ module that is finitely generated and projective as an A-module. Let $Q$ denote the $A^{G}$-G-module $P / \Delta_{G}(A) P$.

(a) $P \cong A \otimes_{A^{C}} Q$ ( $A$-G-module isomorphism $)$.

(b) The $\lambda$-isotopic component $Q_{\lambda}$ of $Q$ is isomorphic as an $A^{G}$-G-module to $H_{\lambda} \otimes_{k} V_{\lambda}$, where $H_{\lambda}=\operatorname{Hom}_{k-G}\left(V_{\lambda}, Q\right)$, a projective $A^{G}$-module with trivial G-action. Hence $P$ is isomorphic as an $A$-G-module to $\oplus_{\lambda} A \otimes_{A^{c}} H_{\lambda} \otimes_{k} V_{\lambda}$.

(c) Suppose that projective $A^{G}$-modules are free. (This is automatic if projective $A$-modules are free.)

Then $P$ is $A$-G-isomorphic to $W_{A}=A \otimes_{k} W$ for some $k$-G-module $W$.

Assertion (a) follows from Corollary (6.4), and (b) follows then from (a) and the discussion preceding Theorem (3.1). If projective $A^{G}$-modules are free, then we have, for each $\lambda \in \Lambda, H_{\lambda} \cong\left(A^{G}\right)^{n_{\lambda}}$ for some $n_{\lambda} \geqslant 0$. Hence $A \otimes_{A^{c}} H_{\lambda} \otimes_{k} V_{\lambda} \cong A \otimes_{k}$ $V_{\lambda}^{n_{\lambda}}$, so $P \cong A \otimes_{k} W$, where $W=\oplus_{\lambda} V_{\lambda}^{n_{\lambda}}$. Finally, the parenthetical assertion of (c) follows from:

(10.2) Remark. If $B \stackrel{i}{\rightarrow} C \stackrel{p}{\rightarrow} B$ is a retraction of rings, $p \circ i=\operatorname{Id}_{B}$, and if projective $C$-modules are free, then every projective $B$-module $P$ is free. Indeed $P \cong B \otimes_{C, p}\left(C \otimes_{B, i} P\right)$, and $C \otimes_{B, i} P$ is a free $C$-module.

Geometric interpretation of (10.1). Every $G$-vector bundle on $X$ is the pullback via $\eta: X \rightarrow X / G$ of one on $X / G$. If vector bundles on $X / G$ are trivial, then every $G$-vector bundle on $X$ is isomorphic to $X \times W$ for some $k$ - $G$-module $W$.

(10.3) Theorem. Assume that:

(a) The action of $G$ on $X=\operatorname{Spec}(A)$ is fix pointed.

(b) $X^{G}$ is a local complete intersection in $X$. (This is automatic if $X^{G} \subset X_{\mathrm{reg}}$, by Proposition (8.4).) 
Then $\pi: X / G$ admits the structure of a G-vector bundle over $X / G$. As such,

$$
X \cong \bigoplus_{\lambda \in \Lambda}\left(E_{\lambda} \otimes_{k} V_{\lambda}\right)
$$

(vector bundle direct sum), where $E_{\lambda}$ for each $\lambda \in \Lambda$ is a vector bundle over $X / G$ with trivial G-action.

The first assertion follows the Normal Linearization Theorem (9.1), with $X^{G}$ ( $\cong X / G)$ in the role of $X_{0}$. The second assertion follows then from the beginning of $\S 3$.

(10.4) Corollary. Assume (a) and (b) of Theorem (10.2) as well as:

(c) All vector bundles on $X / G$ are trivial. (This is automatic if all vector bundles on $X$ are trivial; Remark (10.2).)

Then $X$ is isomorphic as a $G$-scheme to $(X / G) \times W$, where $W$ is a $k$-G-module on which the action of $G$ is one fix pointed.

With the notation of Theorem (10.3) we have, for each $\lambda \in \Lambda, E_{\lambda} \cong(X / G) \times k^{n_{\lambda}}$ for some $n_{\lambda} \geqslant 0$, so $E_{\lambda} \otimes_{k} V_{\lambda} \cong(X / G) \times V_{\lambda}^{n_{\lambda}}$. It follows that $X \cong(X / G) \times W$, where $W=\oplus_{\lambda} V_{\lambda}^{n_{\lambda}}$. Clearly $W / G$ must be a single point, so the origin is the unique closed $G$-orbit in $W$, whence the last assertion.

(10.5) CoRollary. Suppose that the reductive group $G$ acts on the affine space $X=\mathbf{A}_{K}^{n}$. If the action is fix pointed, then $X$ is G-isomorphic to $Y \times \mathbf{A}_{k}^{m}$, where $Y=X^{G} \cong X / G$, and where $G$ acts linearly and one fix pointedly on $\mathbf{A}_{k}^{m}$. The action of $G$ on $X$ can be linearized iff $Y$ is isomorphic to $\mathbf{A}_{k}^{n-m}$; this is automatic whenever $n-m \leqslant 2$.

Of the assumptions (a), (b), (c) of Corollary (10.4), (a) has been assumed, (b) follows because $X$ is regular (see Proposition (8.4)), and (c) is just the Quillen-Suslin Theorem ("Serre Conjecture") (see $[\mathbf{Q}$ and $\mathbf{S}]$ ). The first assertion thus follows from Corollary (10.4) with $m=\operatorname{dim}_{k}(W)$. If the action of $G$ can be linearized, then $Y=X^{G}$ must be isomorphic to a linear space, hence $Y \cong \mathbf{A}_{k}^{n-m}$. Conversely any isomorphism $Y \cong \mathbf{A}_{k}^{n-m}$ clearly leads to a linear action of $G$ on $Y \times \mathbf{A}_{k}^{m} \cong \mathbf{A}_{k}^{n-m} \times$ $\mathbf{A}_{k}^{m} \cong \mathbf{A}_{k}^{n}$. If $n-m \leqslant 2$, then $Y \cong \mathbf{A}_{k}^{n-m}$ by the Cancellation Theorem of Fujita, Miyanishi and Sugie (cf. [Ru]).

(10.6) Corollary. If the action of $G$ on $X$ is one fix pointed and the fixed point $x$ is regular in $X$, then $(X, x)$ is $G$-isomorphic to the tangent space action on $\left(T_{x}(X), 0\right)$.

This is (10.4) in the case when $X / G$ is a point.

(10.7) COROLlaRY. If the reductive group $G$ acts on the affine scheme $X=\operatorname{Spec}(A)$ with a dense orbit and a regular fixed point $x$, then $(X, x)$ is $G$-isomorphic to the tangent space action on $\left(T_{x}(X), 0\right)$.

By Corollary (10.6) it suffices to check that the action is one fix pointed. The hypotheses clearly imply that $\{x\}$ is the unique closed orbit, so $X / G$ is a point, and $A^{G}$ is an artin local ring. If $A^{G}=k$, i.e. if $A^{G}$ is reduced, then $\Delta_{G}(A)=m_{x}$, and the 
action is one fix pointed, as required. Suppose, on the contrary, that $a \in A^{G}$ is nilpotent,$\neq 0$. Then $\operatorname{ann}_{A}(a)$ is a $G$-stable ideal $\neq A$, hence contained in the unique maximal $G$-stable ideal $m_{x}$. Hence $a$ remains $\neq 0$ in $A_{m_{x}}$. But this contradicts the assumed regularity of $A_{m_{x}}$.

(10.8) Remarks. Corollary (10.7) is contained in Luna [L, III, §1, Corollaire 2]. Note that it furnishes an equivariant characterization of affine spaces, as varieties admitting a reductive group action with a dense orbit and a regular fixed point.

11. Examples of fix pointed actions. We consider a reductive group $G$ acting on $X=\operatorname{Spec}(A)$, with an eye to recognizing when the action is fix pointed.

(11.1) EXAMPLE. Supposes that $G$ is diagonalizable, i.e. isomorphic to a subgroup of a torus. Consider the character group $G^{v}=\operatorname{Hom}\left(G, \mathbf{G}_{m}\right)$, which we shall write additively; if $\chi \in G^{v}$ we write $t^{\chi}$ in place of $\chi(t)$. The action of $G$ on $X$ is equivalent to the $G^{v}$-graded ring structure,

$$
A=\bigoplus_{\chi \in G^{v}} A_{\chi},
$$

where $A_{\chi}$ consists of semi-invariants of weight $\chi$ for the (translation) action of $G$ on $A$. We have $A_{0}=A^{G}$, and $\Delta_{G}(A)=\oplus_{\chi \neq 0} A_{\chi}$. In order for the action to be fix pointed, i.e. in order for $\Delta_{G}(A)$ to be an ideal, it is clearly necessary and sufficient that $A_{\chi} \cdot A_{-\chi}=0$ for all $\chi \neq 0$ in $G^{v}$.

Suppose that $A$ is an integral domain. Then $\Sigma \stackrel{\text { def }}{=}\left\{\chi \in G^{v} \mid A_{\chi} \neq 0\right\}$ is clearly a submonoid of $G^{v}$. In this case the action is fix pointed iff

$$
\chi,-\chi \in \Sigma \Rightarrow \chi=0,
$$

i.e. $\Sigma$ contains no nontrivial invertible elements. This is the condition which Kambayashi and Russell [KR] call "unmixedness" (when $G$ is a torus). Theorem (10.3) above thus becomes, when $G$ is a torus and $X$ is regular, a theorem of Kambayashi and Russell [KR, Theorem (2.5)], describing $A$ as a $\Sigma$-graded symmetric algebra over $A^{G}=A_{0}$. Their Theorem (3.4) is similarly subsumed in Corollary (10.5) above.

(11.2) Proposition. Let $B \subset A$ be a finitely generated G-stable subalgebra, and let $C=A / J$ where $J$ is a $G$-stable ideal of $A$. If the action of $G$ on $A$ is fix pointed, then the actions of $G$ on $B$ and $C$ are fix pointed.

Indeed since $\Delta_{G}$ is an exact functor on a $k$-G-module we have $\Delta_{G}(B)=B \cap$ $\Delta_{G}(A)$, an ideal of $B$, and $\Delta_{G}(C)=$ the image $\bmod J$ of $\Delta_{G}(A)$, an ideal of $C$.

(11.3) Proposition. Let $x \in X_{\text {reg }}$ be a regular fixed point of $G$, and let $T=T_{x}(X)$, the tangent space at $x$. If $G$ acts fix pointedly on $X$, then $G$ acts fix pointedly on $T$. The converse holds if $A$ is an integral domain.

We can write $m_{x}=T^{*} \oplus m_{x}^{2}$, where $T^{*}$ is a $G$-submodule of $m_{x}$, isomorphic to the dual of $T$. Let $S$ denote the symmetric algebra $S_{k}\left(T^{*}\right)$, and $S_{+}=T^{*} \cdot S$. The inclusion $T^{*} \subset A$ defines a $G$-equivariant homomorphism $S \rightarrow A$ which, since $x$ is regular, induces an isomorphism from $\operatorname{gr}_{S_{+}}(S)=S$ to $\operatorname{gr}_{m_{x}}(A)$. Since $\bigcap_{n \geqslant 0} S_{+}^{n}=0$ it 
follows that $S \rightarrow A$ is injective, so $G$ acts fix pointedly on $S$ if it does so on $A$ by Proposition (11.2). Suppose that $G$ acts fix pointedly on $S$. Then, again by (11.2), it does so also on $S / S_{+}^{n} \cong A / m_{x}^{n}=A_{n}$. Thus $A_{n}=A_{n}^{G} \oplus \Delta_{G}\left(A_{n}\right)$ with $\Delta_{G}\left(A_{n}\right)$ an ideal. Passing to the $m_{x}$-adic completion $\hat{A}=\operatorname{dim}_{n} A_{n}$ we have $\hat{A}=\hat{A}^{G} \oplus \widehat{\Delta_{G}(A)}$, with $\widehat{\Delta_{G}(A)}$ an ideal. If $A$ is an integral domain then $\bigcap_{n \geqslant 0} m_{x}^{n}=0$ so $A \rightarrow \hat{A}$ is injective, and hence $\Delta_{G}(A)=A \cap \widehat{\Delta_{G}(A)}$ is an ideal of $A$.

In view of (11.3) it is natural to ask when a linear action is fix pointed.

(11.4) Proposition. Let $W$ be a finite dimensional $k$-G-module, $W=W^{G} \oplus$ $\Delta_{G}(W)$. The following conditions are equivalent.

(a) The action of $G$ on $W$ is fix pointed.

(b) The action of $G$ on $\Delta_{G}(W)$ is (one) fix pointed.

(c) $S_{k}\left(\Delta_{G}(W)^{*}\right)^{G}=k$.

We have

$$
S_{k}\left(W^{*}\right)=S_{k}\left(\left(W^{G}\right)^{*}\right) \otimes_{k} S_{k}\left(\Delta_{G}(W)^{*}\right)
$$

and

$$
\Delta_{G}\left(S_{k}\left(W^{*}\right)\right)=S_{k}\left(\left(W^{G}\right)^{*}\right) \otimes_{k} \Delta_{G}\left(S_{k}\left(\Delta_{G}(W)^{*}\right)\right),
$$

whence $(\mathrm{a}) \Leftrightarrow$ (b) (since $\left.\Delta_{G}(W)^{G}=\{0\}\right)$. Since $\Delta_{G}^{+}\left(S_{k}\left(\Delta_{G}(W)^{*}\right)\right)=\Delta_{G}(W)^{*}$. $S_{k}\left(\Delta_{G}(W)^{*}\right)$ we have (b) $\Leftrightarrow$ (c).

(11.5) Proposition. Let $W$ be a finite dimensional $k$-G-module with $W^{G}=0$. Assume that $G$ is diagonalizable, and let $\chi_{1}, \ldots, \chi_{n} \in G^{\prime \prime}$ be the weights of $G$ in $W$ $(n=\operatorname{dim} W$ ). The action of $G$ on $W$ is (one) fix pointed iff

$$
\left.\begin{array}{c}
\sum_{i=1}^{n} a_{i} \chi_{i}=0 \\
\left(a_{i} \in \mathbf{Z}, a_{i} \geqslant 0\right)
\end{array}\right\} \Rightarrow a_{i}=0 \quad \text { for } i=1, \ldots, n .
$$

Let $t_{1}, \ldots, t_{n}$ be a basis of $W^{*}$ with $t_{i}$ semi-invariant of weight $-\chi_{i}$. If $a=$ $\left(a_{1}, \ldots, a_{n}\right) \in \mathbf{Z}^{n}, \quad a_{i} \geqslant 0$, then $t^{a}=t_{1}^{a_{1}} \cdots t_{n}^{a_{n}}$ is semi-invariant of weight $-\sum_{i=1}^{n} a_{i} \chi_{i}$. Hence $(*)$ is equivalent to the condition that $S_{k}\left(W^{*}\right)^{G}=k$, whence the proposition, by $(11.4)(\mathrm{c})$.

(11.6) Corollary (Bialynicki - Birula). If an $n$-dimensional torus $G$ acts effectively on $X=\mathbf{A}_{k}^{n}$, then the action can be linearized.

According to [B-B1, Theorem 1], $G$ has a fixed point, which we may assume to be the origin. The tangent space representation at 0 is faithful, so its weights generate $G^{\prime \prime}$, hence they are linearly independent so, by (11.5) and (11.3), the action of $G$ on $X$ is (one) fix pointed. The corollary thus follows from Corollary (10.6).

(11.7) Proposition. Let $W$ be a finite dimensional $k$-G-module with $W^{G}=0$. Assume that $G$ is semisimple. The action of $G$ on $W$ is (one) fix pointed iff $G$ has a dense orbit in $W$. 
If $G$ has a dense orbit in $W$ then $S_{k}\left(W^{*}\right)^{G}=k$, clearly, so the action is one fix pointed by (11.4)(c). Suppose, conversely, that $S_{k}\left(W^{*}\right)^{G}=k$. Then $S_{k}\left(W^{*}\right)^{G^{0}}=k$, where $G^{0}$ is the identity component of $G$. Since $G^{0}$ has no rational characters and $S_{k}\left(W^{*}\right)$ is factorial it follows from Luna [L1, III, §4], that the maximum dimension of a $G^{0}$-orbit in $W$ is $\operatorname{dim} W-\operatorname{dim} W / G=\operatorname{dim} W$, whence the existence of a dense $G^{0}$-orbit in $W$.

(11.8) Remark. For certain semisimple groups $G$, the $k$ - $G$-modules with a dense orbit have been explicitly determined (cf. [Se]).

(11.9) Remark. Let $G$ be a reductive group acting on $X=\operatorname{Spec}(A)$, with $A$ an integral domain. Put $m=\max _{x \in X} \operatorname{dim} G x$ and $X_{\max }=\{x \in X \mid \operatorname{dim} G x=m\}$. Results of Rosenlicht [Ro] imply that $X_{\max }$ is open dense in $X$. The action is said to be stable (in the sense of Popov [Po]) if there is an open dense set of closed orbits, hence an open dense part of $X_{\max }$ consisting of closed orbits.

Consider the projection $\pi: X \rightarrow X / G$. Let $\xi \in X / G$, and suppose that $\pi^{-1}(\xi)$ is a closed $m$-dimensional orbit. It is disjoint from the $G$-stable closed set $X-X_{\max }$ so there is an $f \in A^{G}$ such that $f=1$ on $\pi^{-1}(\xi)$ and $f=0$ on $X-X_{\max }$. Hence $\pi\left(X-X_{\max }\right)$ is contained in the zero locus of $f$ in $X / G$, so $\xi \notin \overline{\pi\left(X-X_{\max }\right)}$. Thus the complement of $\pi^{-1}\left(\pi\left(\overline{X-X_{\max }}\right)\right)$ is an open nonempty, hence dense, set consisting of closed orbits. In conclusion, the action is stable iff there is a single closed orbit in $X_{\max }$.

Suppose further that $G$ has no rational characters (e.g. that $G$ is connected and semisimple) and that $X$ is factorial. Then Popov [Po] has shown that the action is stable iff an open dense part of $X_{\max }$ consists of affine orbits. Thus the action is not stable iff no orbit in $X_{\max }$ is closed, and most orbits in $X_{\max }$ are nonaffine.

(11.10) Proposition. If $G=\mathrm{SL}_{2}$ acts on $X=\operatorname{Spec}(A)$ where $A$ is factorial, and if the action is not stable, then the action is fix pointed.

Define $h: k^{x} \rightarrow G$ and $u: k \rightarrow G$ by

$$
h(a)=\left(\begin{array}{cc}
a & 0 \\
0 & a^{-1}
\end{array}\right) \text { and } u(b)=\left(\begin{array}{ll}
1 & b \\
0 & 1
\end{array}\right) .
$$

Put $T=h\left(k^{x}\right), T_{n}=\left\{h(a) \mid a^{n}=1\right\}, U=u(k), B=T \cdot U, B_{n}=T_{n} \cdot U$, and $N=$ $\langle T, w\rangle, w=\left(\begin{array}{cc}0 & -1 \\ 1 & 0\end{array}\right)$. Up to conjugacy the closed subgroups $H$ of $G$ are: finite, $B_{n}$ $(n \geqslant 1), T, N, B, G$. The corresponding quotients $G / H$ are affine except for $G / B \cong \mathbf{P}^{1}$, and $G / B_{n}$, which are quasi-affine.

If $m=\max _{x \in X} \operatorname{dim} G x$ is 3 the action is stable by Popov's result quoted above. Suppose that the action is not stable, hence $m \leqslant 2$. Let $x \in X$. Then $\operatorname{dim} G x=0$ or 2. If $\operatorname{dim} G x=2$ then, since $G x$ is not closed (by the discussion above), $\overline{G x}$ must contain lower dimensional orbits, hence fixed points. It follows, as claimed, that the action is fix pointed (see (10.0)).

(11.11) Corollary (Panyushev [P]). If $G=\mathrm{SL}_{2}$ acts on $X=\mathbf{A}^{n}$ and the action is not stable, then $X$ is G-isomorphic to $(X / G) \times V$ for some $k$-G-module $V$.

This follows from Proposition (11.10) and Corollary (10.5). 
12. An equivariant approach to the Jacobian Conjecture. An endomorphism $\varphi$ : $\mathbf{A}_{\mathbf{C}}^{n} \rightarrow \mathbf{A}_{\mathbf{C}}^{n}, \varphi(X)=\left(\varphi_{1}(X), \ldots, \varphi_{n}(X)\right)$, defines an endomorphism of the polynomial algebra $\mathbf{C}[X]=\mathbf{C}\left[X_{1}, \ldots, X_{n}\right], \bar{\varphi}: X_{i} \mapsto \varphi_{i}(X)$, sending $f(X)$ to $f(\varphi(X))$. The Jacobian matrix is denoted

$$
\varphi^{\prime}(X)=\left(\begin{array}{ccc}
D_{1} \varphi_{1} & \cdots & D_{n} \varphi_{1} \\
\vdots & & \vdots \\
D_{1} \varphi_{n} & \cdots & D_{n} \varphi_{n}
\end{array}\right),
$$

where $D_{i}=\partial / \partial X_{i}$. The Chain Rule takes the form

$$
(\psi \circ \varphi)^{\prime}(X)=\psi^{\prime}(\varphi(X)) \cdot \varphi^{\prime}(X) .
$$

If $\varphi$ is invertible and $\psi=\varphi^{-1}$, i.e. $\psi(\varphi(X))=X$, then (1) shows that $\varphi^{\prime}(X)^{-1}$ is invertible, and

$$
\varphi^{\prime}(X)^{-1}=\left(\varphi^{-1}\right)^{\prime}(\varphi(X)) .
$$

The Jacobian Conjecture asserts, conversely, that if $\varphi^{\prime}(X)$ is invertible, then $\varphi$ is invertible (cf. [BCW]). Suppose that $\varphi^{\prime}(X)$ is invertible. Modifying $\varphi$ by a translation, if necessary, we may further assume that $\varphi(0)=0$. Then at least $\varphi$ has a local analytic inverse near 0 , say $\varphi^{-1}(X)=\left(\varphi_{1}^{-1}(X), \ldots, \varphi_{n}^{-1}(X)\right)$ with each $\varphi_{i}^{-1}(X)$ in the ring $\mathbf{C}\{X\}=\mathbf{C}\left\{X_{1}, \ldots, X_{n}\right\}$ of convergent power series. We can view $\bar{\varphi}: X_{i} \mapsto \varphi_{i}(X)$ as an automorphism of $\mathbf{C}\{X\}$, with inverse $\bar{\varphi}^{-1}: X_{i} \mapsto \varphi_{i}^{-1}(X)$.

Let $D$ be a derivation of $\mathbf{C}\{X\}$. Then $\bar{\varphi} \circ D \circ \bar{\varphi}^{-1}$ is another such derivation, sending $X_{i}$ to

$$
\begin{aligned}
{\left[\left(\bar{\varphi} \circ D \circ \bar{\varphi}^{-1}\right)\left(X_{i}\right)\right](X) } & =D\left(\varphi_{i}^{-1}\right)(\varphi(X)) \\
& =\left(\sum_{j=1}^{n} D_{j}\left(\varphi_{i}^{-1}\right) \cdot D\left(X_{j}\right)\right)(\varphi(X)) \\
& =\sum_{j=1}^{n} D_{j}\left(\varphi_{i}^{-1}\right)(\varphi(X)) \cdot D\left(X_{j}\right)(\varphi(X)) .
\end{aligned}
$$

Now by (2) above, $D_{j}\left(\varphi_{i}^{-1}\right)(\varphi(X))$ is the $(i, j)$-entry of $\varphi^{\prime}(X)^{-1}$, and hence, by the assumed invertibility of $\varphi^{\prime}(X)$, is a polynomial. Supposing further that $D$ is a polynomial derivation, i.e. that each $D\left(X_{j}\right)$ is a polynomial, then $D\left(X_{j}\right)(\varphi(X))$ is likewise a polynomial. In conclusion:

If $\varphi: \mathbf{A}_{\mathbf{C}}^{n} \rightarrow \mathbf{A}_{\mathbf{C}}^{n}$ has invertible Jacobian $\varphi^{\prime}(X)$, then for every polynomial derivation $D$ of $\mathbf{C}[X], \bar{\varphi} \circ D \circ \bar{\varphi}^{-1}$ (which is a priori only a derivation of $\mathbf{C}\{X\})$ is again a polynomial derivation.

Continuing to assume that $\varphi^{\prime}(X)$ is invertible and that $\varphi(0)=0$, consider now the group $G=\mathrm{SL}_{n}(\mathbf{C})$ with its natural (linear) action on $\mathbf{A}_{\mathbf{C}}^{n}$. This is a one fix pointed action with a dense orbit. We can view $\varphi^{-1} G \varphi$ as a group of germs of automorphisms near 0 of $\mathbf{A}_{\mathbf{C}}^{n}$, and $\bar{\varphi} \bar{G} \bar{\varphi}^{-1}$ as a group of automorphisms of $\mathbf{C}\{X\}$. Suppose that $\varphi^{-1} G \varphi$ actually consists of polynomial automorphisms, i.e. that $\bar{\varphi} \bar{G} \bar{\varphi}^{-1}$ stabilizes $\mathbf{C}[X]$. Then $\varphi^{-1} G \varphi$ defines a new action of $G$ on $\mathbf{A}_{\mathbf{C}}^{n}$, and $\varphi$ becomes a 
$G$-morphism from $\mathbf{A}_{\mathbf{C}}^{n}$ with the new action to $\mathbf{A}_{\mathbf{C}}^{n}$ with the natural action. As such it is a $G$-linear approximation to the new action, in the sense of Remark (9.2)(4), and the Normal Linearization Theorem (9.1) implies then that $\varphi$ is an isomorphism.

To find reasons for $\varphi^{-1} G \varphi$ to consist of polynomial automorphisms, consider the Lie algebra $\mathfrak{g}=\mathfrak{S} \mathfrak{l}_{n}(\mathbf{C})$ of $G$. It acts naturally as a Lie algebra of (linear) derivations of $\mathbf{C}[X]$. The differential of the new action, $\varphi^{-1} G \varphi$, of $G$, represents $g$ as the algebra $\bar{\varphi} \mathfrak{g} \bar{\varphi}^{-1}$ of derivations of $\mathbf{C}\{X\}$. However observation (3) above tells us that $\bar{\varphi} \mathfrak{g} \bar{\varphi}^{-1}$ is in fact again a Lie algebra of polynomial derivations. This gives a representation of the Lie algebra $\mathfrak{g}$ as derivations of $\mathbf{C}[X]$ which, on the subalgebra $\mathbf{C}[\varphi]=$ $\mathbf{C}\left[\varphi_{1}, \ldots, \varphi_{n}\right]$, looks like the natural representation in the $\varphi$-coordinates. Then $\bar{\varphi} \bar{G} \bar{\varphi}^{-1}$ is the exponential of this polynomial representation of $\mathfrak{g}$. In order to show then that $\bar{\varphi} \bar{G} \bar{\varphi}^{-1}$ is polynomial, it suffices to show that the action of $\bar{\varphi} \mathfrak{g} \bar{\varphi}^{-1}$ on $\mathrm{C}[X]$ is locally finite, i.e. that $\mathbf{C}[X]$ is a union of finite dimensional subspaces stable under $\bar{\varphi} \mathfrak{g} \bar{\varphi}^{-1}$. For this will then imply that the nilpotent elements $t X_{i} D_{j}(i \neq j)$ in $\mathfrak{g}$ yield locally nilpotent derivations $\bar{\varphi} \cdot t X_{i} D_{j} \cdot \bar{\varphi}^{-1}$ of $\mathrm{C}[X]$, and hence polynomial automorphisms $\exp \left(\bar{\varphi} \cdot t X_{i} D_{j} \cdot \bar{\varphi}^{-1}\right)=\bar{\varphi} \cdot \exp \left(t X_{i} D_{j}\right) \cdot \bar{\varphi}^{-1}$. The elementary matrices $\exp \left(t X_{i} D_{j}\right)$ generate $G=\mathrm{SL}_{n}(\mathbf{C})$, so we conclude that $\varphi^{-1} G \varphi$ is polynomial, as desired.

In conclusion, the Jacobian Conjecture (that $\varphi$ is an isomorphism) would follow if one could show that the Lie algebra $\bar{\varphi} \mathfrak{g} \mathfrak{l}_{n}(\mathbf{C}) \bar{\varphi}^{-1}$ of derivations of $\mathbf{C}[X]$ acts locally finitely on $\mathbf{C}[X]$. Hopefully the (well-studied) representation theory of $\mathfrak{s l}_{n}(\mathbf{C})$ may be of some help in this regard.

\section{REFERENCES}

[BCW] H. Bass, E. H. Connell and D. Wright, The Jacobian Conjecture: Reduction of degree and formal expansion of the inverse, Bull. Amer. Math. Soc. (N.S.) 7 (1982), 287-330.

[B-B1] A. Bialynicki-Birula, Remarks on the action of an algebraic torus on $k^{n}$, Bull. Acad. Polon. Sci. Ser. Math. Astronom. Phys. 14 (1966), 177-181.

[B-B2] __ Remarks on the action of an algebraic torus on $k^{n}$. II, Bull. Acad. Polon. Sci. Ser. Math. Astronom. Phys. 15 (1967), 123-125.

[NB1] N. Bourbaki, Algébre commutative, Chapitres 1 et 2, Hermann, Paris, 1961.

[NB2] _ Algébre commutative, Chapitres 8 et 9, Masson, Paris, 1983.

[EGA] A. Grothendieck, Éléments de géométrie algébrique. IV (Seconde partie), Publ. Inst. Hautes Etude Sci. No. 24, 1965.

[J] H. W. E. Jung, Über ganze birationale Transformationen der Ebene, J. Reine Angew. Math. 184 (1942), 161-174.

[K] T. Kambayashi, Automorphism group of a polynomial ring and algebraic group action on an affine space, J. Algebra 60 (1979), 439-451.

[KR] T. Kambayashi and P. Russell, On linearizing algebraic torus actions, J. Pure Appl. Algebra 23 (1982), 243-250.

[KP] H. Kraft, Algebraic groups, invariants and representations, International Conference, Trento, Italy, 1981, pp. 1.6-6.6.

[L] D. Luna, Slices étales, Bull. Soc. Math. France Mém. 33 (1973), 81-105.

[N] M. Nagata, Lectures on the fourteenth problem of Hilbert, Tata Inst. of Fundamental Research, Bombay, 1965.

[P] D. I. Panyushev, Semisimple automorphism groups of four dimensional affine space, Izv. Akad. Nauk SSSR 47 (1983), 881-894. (Russian)

[Po] V. L. Popov, On the stability of the action of an algebraic group on an algebraic variety, Math. USSR-Izv. 6 (1972), 367-379.

[Q] D. Quillen, Projective modules over polynomial rings, Invent. Math. 36 (1976), 167-171. 
[R1] R. Richardson, The conjugating representation of a semisimple algebraic group, Invent. Math. 54 (1979), 229-245.

[R2] _ An application of the Serre conjecture to semisimple algebraic groups, Algebra (Carbondale 1980), Lecture Notes in Math., vol. 848, Springer-Verlag, Berlin and New York, pp. 141-151.

[Ro] M. Rosenlicht, Some basic theorems on algebraic groups, Amer. J. Math. 78 (1956), 401-443.

[Ru] P. Russell, On affine-ruled rational surfaces, Math. Ann. 255 (1981), 287-302.

[Se] F. J. Servedio, Prehomogeneous vector spaces and varieties, Trans. Amer. Math. Soc. 176 (1973), 421-444.

[Sh] I. Shafarevič, Some infinite dimensional algebraic groups, Rend. Mat. (5) 25 (1966), 208-212.

[Sp] T. A. Springer, Invariant theory, Lecture Notes in Math., vol. 585, Springer-Verlag, Berlin and New York, 1977.

[S] A. Suslin, Projective modules over a polynomial ring, Dokl. Akad. Nauk SSSR 26 (1976). (Russian)

Department of Mathematics, Columbia University, New York, New York 10027

Department of Mathematics, State University of New York at Albany, Albany, New York 12222 